Brigitte Young

\title{
Genderregime und Staat in der globalen Netzwerk-Ökonomie
}

\section{Staat und Genderforschung}

Es ist in vielfacher Hinsicht paradox, daß just in dem Moment, in dem die feministische Forschung und Praxis den Staat nicht mehr als Hochburg des Patriarchats ablehnt ${ }^{1}$ und sogar den patriarchalen Wohlfahrtsstaat verteidigt, der Staat seine Souveränität nach innen und außen zunehmend verliert (Strange 1996; Mayntz 1997). Die feministische Annährung an den Staat in den 80er Jahren einerseits und die Unterminierung staatlicher Steuerungsfähigkeit andererseits sind mit der von Margaret Thatcher und Ronald Reagan eingeleiteten ideologischen »Wende vom Keynesianismus zum Neoliberalismus" verbunden. Die Entwicklung in Richtung einer reinen und perfekten Marktwirtschaft - der »neoliberalen Utopie« (Bourdieu 1998) verbunden mit einer Abwertung aller kollektiven Solidaritäts- und Zugehörigkeitsgefühle ${ }^{2}$ hat - wenn nicht zu einer allgemeinen Politisierung der Frauenbewegung - jedoch zu einer zunehmenden Unsicherheit in ihren Reihen - beigetragen. Dieser neo-liberale »backlash« (Faludi 1991) gegen die durchaus noch dürftigen Errungenschaften von politischer und ökonomischer Gleichstellung der Geschlechter in westlich-kapitalistischen Ländern hat die feministische Forschung zunehmend auf die Transformation von Staatlichkeit im Kontext der ökonomischen Globalisierung gelenkt.

Im internationalen feministischen Diskurs lassen sich zwei wichtige Strömungen unterscheiden: einerseits der weitverbreitete anglo-amerikanische und skandinavische $»$ Staatsfeminismus« (state feminism) ${ }^{3}$ (vgl. dazu Stetson/

1 Die feministische Ablehnung des Staates wird keineswegs von allen Wissenschaftlerinnen geteilt. Die Distanzierung vom Staat ist ein besonderes Merkmal der deutschen autonomen Frauenbewegung, die erst Mitte der 80er Jahre eine vorsichtige Annäherung an den Staat vollzogen hat (vgl. Lang 1997; Kontos 1990; Kulawik 1991/92). Skandinavierinnen, Australierinnen und auch ein Teil der US-Amerikanischen liberalen Frauenbewegung waren im Vergleich immer schon »staatsfreundlicher« und haben gezielt den »Marsch durch die Institutionen gewählt « (state feminism), um die gesellschaftliche Situation der Frauen politisch zu verändern (Stetson and Mazur 1995).

2 Baroness Thatcher erklärte, daß es für sie keine Gesellschaft gäbe, sondern nur Individuen.

3 Hester Eisenstein spricht von den »femocrats « in staatlichen Bürokratien und Institutio-

PROKLA. Zeitschrift für kritische Sozialwissenschaft, Heft 111, 28. Jg. 1998, Nr.2, 175-198 
Mazur 1995; Eisenstein 1990; Sassoon 1987) und andererseits die im deutschsprachigen Raum vorherrschende Diskussion über das »Männerbündische« im Staat (Kreisky/Sauer 1997; Kulawik/Sauer 1996; Kerchner/Wilde 1996). Ziel dieser Strategie ist es, den männlichen Staat zu »entgeschlechtlichen « und dann durch feministische Intervention zu »vergeschlechtlichen«. Obwohl die Diskurse, Praktiken und Strategien dieser feministischen staatstheoretischen Diskussionen sich erheblich voneinander unterscheiden, bleiben beide Ansätze der Konzeption des klassischen National- und Wohlfahrtstaates verhaftet (vgl. Young 1997; Demirovic/Pühl 1997).

Die nationalstaatliche Fokussierung der feministischen Staatstheorie muß einerseits als Resultat der von vielen Frauen wahrgenommenen Privatisierung öffentlicher Dienstleistungen und der zunehmenden Reduzierung der öffentlichen Ausgaben verstanden werden. Andererseits ist die demokratische Einflußnahme und Partizipation noch immer auf den nationalstaatlich begrenzten Raum beschränkt. Somit verspricht die im Fordismus zur Blüte gereifte »nationale Schicksalsgemeinschaft« (Held 1991) weiterhin die Vereinbarkeit der bisher in westlichen Demokratien ungleichen Prinzipien von Markt und Demokratie. Diese formal-demokratische Ordnung westlicher Prägung hat durchaus die ökonomische Ungleichheit zwischen (männlichen) Bourgeois und Proletariern durch die Gleichheit der Citoyens abgemildert und soziale Gleichheit durch wohlfahrtsstaatliche Mindeststandards korrigiert (vgl. Altvater 1997; kritisch dazu Pateman 1988; Nelson 1990; Gordon 1990; Fraser 1990; Ostner 1994). Somit ist das feministische Festhalten an der »nationalen Schicksalsgemeinschaft « durchaus verständlich, doch steht die Grundannahme in Frage, ob diese national-staatliche Fokussierung in Zeiten der Globalisierung und der zunehmenden Entgrenzung von Politik nicht zum Anachronismus wird.

Es stellt sich nun die Frage nach der Vereinbarkeit vom grenzenlosen Markt und dem begrenzten Ort der Demokratie und danach wie sich neue Genderregime und -ordnungen in den Netzwerkstrukturen zwischen Markt und Staat konstituieren.

\section{Exkurs: Genderregime im Fordismus}

Im folgenden wird davon ausgegangen, daß die Transformation historisch spezifischer Akkumulationsmodelle mit der Rekonfiguration von Geschlechterregimen und -ordnungen in Zusammenhang steht. Mit dem Begriff $»$ gender « soll zwischen der gesellschaftlichen Konstruiertheit des Geschlechts (soziales Geschlecht) und des biologischen Geschlechts (sex)

nen, die von »innen« eine politische Veränderung zur Gleichstellung der Geschlechter anstreben (Eisenstein 1990). 
unterschieden werden. Linda Gordon definiert gender als eine Reihe von Bedeutungssystemen, die in bezug auf sexuelle Unterschiede und im Kontext eines männlichen Herrschaftssystems gesellschaftlich konstruiert werden (Gordon 1993). Gender bezeichnet somit ein gesamtgesellschaftliches Netzwerk hierarchisch geregelter gesellschaftlicher Beziehungen, die entlang einer geschlechtlichen Trennlinie sozial verortet sind (vgl. Kreisky/ Sauer 1995). Genderregime sind somit als institutionalisierte Geschlechterpraktiken und Formen zu verstehen, die als ein Geflecht von Normen, Regelungen und Prinzipien in den Strukturen gesellschaftlicher Praktiken verankert sind. Genderordnungen sind dann die Verkörperung einer Reihe von diesen institutionalisierten Praktiken, die zusammen eine »macro-politics of gender « ergeben. Nach Connell wird die Genderordnung als ein dynamischer Prozeß - als der gegebene Bezugsrahmen in dieser Makropolitik definiert (Connell 1987: 139, 141). Somit sind Genderregime und Genderordnungen keine statischen Konstellationen, sondern stehen in einem $\gg$ reflexiven Verhältnis« (Giddens 1984) zu den gegebenen Machtverhältnissen der Geschlechter, die sich durch die gesellschaftlichen Interessenkonflikte, die Formierung und Auflösung von akzeptierten Kategorien und die Neuordnung von institutionellen Verhältnissen konstituieren (Connell 1987: 139). Genderregime repräsentieren einerseits eine symbolische Genderordnung und andererseits verkörpern sie eine Arena der Macht, in der die Definition der Geschlechterverhältnisse immer wieder neu erkämpft werden muß. Genderregime implizieren weder die Existenz einer einzigen historischen »Männlichkeit « noch einer unveränderten »Weiblichkeit«, sondern weisen auf den historischen Prozeß der geschlechtsspezifischen Identitätsbildung und asymmetrischen Machtverteilung zwischen den Geschlechtern innerhalb des jeweiligen gesellschaftlichen Kontexts hin.

Mit den Konzepten Genderregime und -ordnung sollen im folgenden die komplexen Zusammenhänge zwischen der Transformation des fordistischen Akkumulationsregimes und der damit verbundenen Restrukturierung der Geschlechterverhältnisse untersucht werden. In diesem Zusammenhang wird auf Einsichten der Regulationsschule zurückgegriffen, die die kapitalistische Produktionsweise als eine - keineswegs zielgerichtete - Abfolge von verschiedenen Akkumulationsmodellen und Regulationsweisen analysiert. Es stellt sich nun die Frage, wie die Transformation des fordistischen Akkumulationsmodells zu einem flexiblen globalen Akkumulationsregime mit der Konstituierung von geschlechtsspezifischen Herrschaftssystemen verbunden ist. ${ }^{4}$

4 Die Frage, wie kapitalistische und geschlechtsspezifische Herrschaftssysteme verbunden sind und aufeinander einwirken, ist auch unter Feministinnen weitgehend offen. Eine abschließende Antwort kann auch im Rahmen dieses Aufsatzes nicht gegeben werden. 
Der Fordismus ist nicht auf ein ökonomisches Modell reduzierbar, sondern muß als ein gesamtgesellschaftliches System verstanden werden (Aglietta 1979). Nach 1945 war der Fordismus durch die Verkopplung tayloristischer Massenproduktion mit Massenkonsumtion gekennzeichnet. Die Voraussetzungen für das gleichgewichtige Wachstum von Arbeitsproduktivität und realen Lohneinkommen der (männlichen) Arbeiter, die zu dem erfolgreichen ökonomischen Wachstum nach 1945 in den westlichen Industrieländern führten, waren die national-territorialen institutionellen Regulationsweisen der Lohn- und Geldverhältnisse. Die Kopplung der Reallöhne an die Produktivität und die nationalstaatliche antizyklische Geld- und Wirtschaftspolitik bildeten die Voraussetzung für die Stabilisierung des wirtschaftlichen Aufschwungs. Historische Phasen eines solchen »virtuous circle« können nur dann stabil sein, wenn die Kompatibilität zwischen Akkumulation und Nachfrage durch entsprechend regulierende staatliche und gesellschaftliche Institutionen die Dynamik des Akkumulationsprozesses ausbalancieren. Eine wichtige Funktion des Keynesianischen Wohlfahrtsstaates war es, vor allem die Nachfrage zu regeln und die sozialen Rechte über neue Normen des Massenkonsums auszuweiten (Jessop 1994). Diese Phase (1950-1973) wird aus dem heutigen Blickwinkel nostalgisch als das »Goldene Zeitalter des Kapitalismus « gesehen.

Die fordistische Industrialisierung ging einher mit der Transformation der extensiven Akkumulation des Agrarkapitalismus und der existierenden Genderregime. Im Zentrum des fordistischen Produktionsmodells stand der weiße Mann sowohl als Manager in den sich herausbildenden neuen vertikalen Organisationsformen von multinationalen Firmen (Chandler 1977) und als Industriearbeiter in der Massenproduktion. Es waren auf den männlichen Arbeiter zugeschnittene Arbeitsplätze und »sein« Familienlohn, den die Tarifverhandlungen zwischen Gewerkschaften und Arbeitgebern bestimmten. Die Löhne und Arbeitsbedingungen wurden in dem von Männern dominierten und vom Staat wenn nicht geschaffenen, so doch anerkannten hierarchischen System der Tarifrunden unter einer begrenzten Anzahl von Interessenverbänden ausgehandelt, welche mit einem beratenden und repräsentativen Monopol für den Prozeß der Entwicklung und Durchführung von Entscheidungen ausgestattet waren (Schmitter 1979).

Diese organisatorischen Strukturen haben einen deutlich geschlechtsspezifischen Charakter. Frauen sind in der Gewerkschaftsführung kaum vertreten, in den Arbeitgeberverbänden, den korporatistischen Verhandlungen, den staatlichen Ministerien und Verwaltungsstrukturen und den $»$ thinktanks « der Wirtschaft kommen Frauen faktisch nicht vor. In diesen Wirtschafts- und Interessenverbänden werden zentrale ökonomische Entscheidungen von gesamtgesellschaftlichen Interesse getroffen. Die Entscheidungen orientieren sich an einem Produktionssystem, das am männlichen In- 
dustriearbeiter ausgerichtet ist. In diesem geschlechtsspezifischen Arrangement sind sich Arbeit und Kapital durchaus einig. Während sie sich einerseits als Klassengegner im kapitalistischen System gegenüberstehen, teilen sie doch andererseits eine gemeinsame Auffassung über die geschlechtsspezifische gesellschaftliche Genderordnung. Die in diesen korporatistischen Machtstrukturen eingeschriebenen asymmetrischen Geschlechterverhältnisse sind die Reflektion der im gesamten Akkumulationsregime vorfindbaren Geschlechterhierarchien.

Das fordistische Akkumulationssystem ist aber nicht nur exklusiv, sondern auch inklusiv gegenüber Frauen. Diejenigen, die in diesem »selektiven Korporatismus « (Esser/Fach 1981) eingeschlossen waren, erlangten materielle Zugeständnisse und ökonomische Sicherheit. Frauen waren zwar nicht aus diesem Kompromiß ausgeschlossen, aber eingeschlossen waren sie nur in ihrem Status als von ihren Ehemännern abhängige »Karrierehausfrauen «. Daß heißt nicht, daß sie auf dem Arbeitsmarkt gar nicht nicht präsent waren. Die Integration der Frauen in den Arbeitsmarkt war durchaus funktional für die Erhöhung des Konsums. Das fordistische Modell beruhte ja gerade auf dem Zugang zur Massenkonsumtion. Die Integration war aber mit der Expansion des Keynesianischen Sozialstaats verbunden und Frauen übten traditionelle soziale Aufgaben aus wie z.B. Altenfürsorge, Kinderbetreuung, Krankenpflege im öffentlichen Dienst und gleichzeitig besetzten sie die niedrigqualifizierten Stellen in den Sozial- und Fürsorgeämtern als Sozialarbeiterinnen. Frauen waren somit in dreifacher Weise mit dem Sozialstaat verbunden: als Angestellte, Klientinnen und als Konsumentinnen der öffentlichen Dienstleistungen. Fast in allen westlichen Industrieländern expandierte der öffentliche Dienstleistungssektor und es waren vor allem Frauen, die in diesem Bereich zu finden waren. Die neugeschaffenen sozialstaatlichen Positionen waren aber keineswegs gleichzusetzen mit der männlichen Karriere eines Facharbeiters. Frauen waren am untersten Ende der beruflichen Hierarchie zu finden, waren meistens schlecht bezahlt und besaßen häufig nur eine niedrige Qualifikation.

Zur selben Zeit öffnete sich für Frauen zunehmend der Einstieg in die untereren Ebenen der Produktionsarbeit. Diese Öffnung war vor allem mit der allgemeinen Dequalifizierung des männlichen Facharbeiters im fordistischen Modell verbunden. Qualifizierte und hochbezahlte Fachkräfte wurden bei der Rationalisierung des Arbeitsprozesses weitgehend überflüssig und Frauen wurden für die niedrigqualifizierten Arbeiten am automatisierten Fließband eingestellt. Daß ihnen der Einzug in die Hallen der männlichen Fabriken gelang, ${ }^{5}$ bedeutete aber nicht, daß der Zugang zu gutbezahl-

5 Frauen waren seit dem Beginn der Industrialisierung in Fabriken als Arbeiterinnen zu finden. Sie füllten auch im Zweiten Weltkrieg die von den Männern freigewordenen Ar- 
ten Industriearbeitsplätzen für sie offen war. Der Arbeitsmarkt blieb geschlechtsspezifisch segregiert. Arbeiterinnen waren in marginalisierten und meist schlecht bezahlten Positionen zu finden, die die geschlechtlich konstruierte gesellschaftliche Arbeitsteilung reproduzierte. Frauen waren somit in niedrigbezahlten und -qualifizierten Jobs segregiert, die von ihnen Geschicklichkeit und Geduld verlangten. Diese geschlechtsspezifische Segregation, die das Genderregime des fordistischen Akkumulationsmodells kennzeichnete, war sowohl in »konservativen « wie in »progressiven« Ländern wie Schweden und Dänemark zu finden. Sie ist Ausdruck einer gesellschaftlichen Ordnung, die auf einer starken Trennung und geschlechtsspezifischen Zuordnung öffentlicher und privater Sphären beruht. Dadurch wurde nicht nur die unbezahlte Hausarbeit entwertet; Frauen wurden trotz ihrer zunehmenden Erwerbstätigkeit dem männlichen Ernährer untergeordnet und ihre Tätigkeit weitgehend als Zusatzverdienst für zusätzliche Konsumbedürfnisse verstanden (Hagen/Jenson 1988; Kurz-Scherf 1996; Young 1998).

Dieses im Fordismus konsolidierte Genderregime kann auf drei zentrale Elemente reduziert werden. Erstens wurde die Rolle der Frau in westlichen Industrieländern - trotz Integration in die Erwerbstätigkeit - weitgehend auf den Reproduktionsbereich zentriert. Zweitens korrespondierte die weibliche Rolle mit der männlichen Rollenzuschreibung als Familienernährer und drittens entstand die geschlechtsspezifische Trennung in Privatheit und Öffentlichkeit als das zentrale Element dieses Genderregimes. In der Geometrie zwischen Klasse und Geschlecht waren Männer direkt dem Markt unterworfen, während Frauen ihren Männern in den Familien und somit nur indirekt dem Markt unterstellt waren.

Die durch die Globalisierung eingeleitete Restrukturierung des Akkumulationsregimes und der damit verbundenen Regulationsweisen stellt die Grenzen der fordistischen Genderordnung erneut zur Disposition. Gleichzeitig zeigt die national-territoriale Eingliederung in die globale Ökonomie, wie ich noch zeigen werde, die zunehmende Schwächung des männlichen Ernährermodells und die damit verbundene geschlechtsspezifische NeuStratifizierung der Genderregime.

\section{Das Neue an der Globalisierung}

»Globalisierung « ist ein offener und widersprüchlicher Prozeß der Umstrukturierung des nationalstaatlich begrenzten fordistischen Akkumulationsregimes und des Keynesianischen Steuerungsystems. Es handelt sich um einen Prozess der Entgrenzung nationaler politischer und ökonomischer

beitsplätze. Mit dem Beginn des Fordismus und der Betonung des männlichen Ernährermodells wurden Frauen immer mehr aus dem Zentrum der Industriearbeit ausgeschlossen und nur selektiv oder temporär integriert. 
Räume. Globalisierung ist aber keineswegs mit einem erreichbaren Ziel (der Globalität) gleichzusetzen genauso wie Modernität als Zustand nicht erreichbar ist (Altvater/Mahnkopf 1996). »Globalität« würde bedeuten, daß wir bereits in einer Weltgesellschaft leben, und zwar in dem Sinne, daß es keine Möglichkeit der Abgrenzung oder Schließung zwischen Staaten, ethnischen Gruppen, Kulturen und Lebensmodellen gäbe. Dieser Zustand einer homogen Weltgesellschaft würde keine lokalen, regionalen oder nationalen Gesellschaften oder Gemeinschaften mehr kennen (Beck 1997). Globalisierung meint demgegenüber den widersprüchlichen Prozeß der Herauslösung (Entbettung) ökonomischer und finanzieller Mechanismen aus national-staatlichen sozialen und politischen Bindungen einerseits und der Entstehung von neuen globalen horizontal-netzwerkförmigen Steuerungssystemen andererseits.

Demnach ist Globalisierung nicht auf einen quantitativen Vergleich von internationalen Handels- und Finanzströmen reduzierbar wie die Kritiker des Globalisierungskonzepts meinen, die die Exportquoten und Direktinvestionen zwischen 1870-1913 zitieren, um die gegenwärtigen globalen Größenordnungen mit dem durchaus vergleichbaren Exportvolumen des imperialen Zeitalters zu relativieren (Hirst/Thompson 1996). Die Internationalisierung vor dem ersten Weltkrieg war eine Austauschbeziehung zwischen Nationalstaaten und geht von Nationen als strategischen Akteuren aus (Strange 1996). Der entscheidende Unterschied zwischen Internationalisierung und Globalisierung ist, daß die ökonomischen und politischen nationalstaatlichen Akteure jetzt der Handlungslogik und den Wettbewerbsbedingungen der globalen Konkurrenz ausgesetzt sind. Der Nationalstaat ist nach wie vor von spezifischer Bedeutung, aber er unterliegt zunehmend der ökonomischen Logik einer globalen Wettbewerbskonkurrenz. Der Nationalstaat wandelt sich zum »Wettbewerbsstaat« (Hirsch 1995). Er wird in erster Linie Akteur der Geoökonomie und erst in zweiter Linie Akteur innerhalb des Systems internationaler Politik (Altvater/Mahnkopf 1996).

Die globale Ökonomie kann zum ersten Mal in der Geschichte als eine Einheit in realer Zeit »on a planetary scale« agieren (Castells 1996: 92). Während die Raum-Zeit-Verhältnisse in der Moderne durch die Trennung der Zeit vom Raum geprägt waren - es war die Erfindung der mechanischen Uhr, die eine einheitliche Dimension »leerer « Zeit zum Ausdruck brachte und die erst eine präzise Einteilung in Raum- und Zeitzonen ermöglichte -, beziehen sich die Raum-Zeit-Verhältnisse in der Globalisierung auf einen »Dehnungsvorgang «, und zwar insoweit, »als die Verbindungsweisen zwischen verschiedenen gesellschaftlichen Kontexten oder Regionen über die Erdoberfläche als Ganze hinweg vernetzt werden « (Giddens 1995: 85). Die vielen Zeiten in unterschiedlichen Kontinenten werden zu einer einzigen Weltzeit zusammengezogen. Dieser Prozeß der Herstel- 
lung einer zeitlichen Einheit trotz ungleichzeitiger Ereignisse erzeugt einen artifiziellen »Weltrhythmus«, der das Virtuelle und das Reale nicht mehr trennbar macht. In dieser virtuellen Realität, die Jean Baudrillard als »Hyperrealität« bezeichnet, leben die Bewohner der ersten Welt in der »Zeit«. Andererseits sind diese Bewohner, die durch die Telekommunikation und die Computertechnik den Globus nur mehr als Dorf wahrnehmen, der räumlichen Dimension enthoben. Bewohner der dritten Welt erfahren die Raum-Zeit-Dimension im umgekehrten Verhältnis zu den Bewohnern der ersten Welt. Sie leben in einem »Raum « der »schwer, unverwüstlich, unberührbar (ist) und der die Zeit festbindet und sich der Kontrolle der Bewohner entzieht« (Bauman 1996: 661).

In dieser »Hyperrealität «, in der die raumzeitlichen Abstandsvergrößerungen zwischen örtlichen und entfernten Ereignissen »gedehnt« werden, entsteht eine komplexe Beziehung zwischen »lokalen Beteiligungsweisen « (der Situation gleichzeitiger Anwesenheit) und der »Interaktion über Entfernung « (den Verbindungen zwischen Abwesenheit und Anwesenheit (Giddens 1995: 85). Mit der Globalisierung tritt demnach eine Intensivierung weltweiter sozialer Beziehungen zwischen lokalen, örtlichen und entfernten sozialen Ereignissen in Kraft. Daraus ergibt sich auch der Widerspruch, »daß sich mit der Wirtschaft auch alles andere globalisiert, und auch das, was gar nicht in der kapitalistischen Form globalisierbar ist « (Altvater/Mahnkopf 1996: 13). Dem Globalisierungsprozeß sind auch immer wieder Schranken gesetzt. Nicht alles, was den Globalisierungstendenzen ausgesetzt ist, kann globalisiert werden. Globalisierung beschränkt sich daher nicht nur auf die Dehnungen und Durchbrechungen von politischen, ökonomischen, sozialen und ökologischen Grenzen, es geht auch um die Gegentendenzen, jene der Begrenzung der Globalisierung.

\section{4. »Informationeller Kapitalismus $\aleph^{6}$ und die Herausbildung von Netzwerkstrukturen}

Die Globalisierungsliteratur reduziert vielfach die neue Produktionsweise auf eine Transformation von der industriellen Produktion hin zu einer Tertiarisierung der Ökonomie. Das Zahlenmaterial läßt diese Interpretation durchaus zu. Nicht nur in den USA nimmt der Anteil des Dienstleistungssektors am Bruttoinlandsprodukt bereits über 70\% ein. Auch in anderen Industrieländern steigt der Anteil der Dienstleistungen während derjenige der industriellen Produktion abnimmt (Thurow 1997). Daraus wird eine lineare Abfolge von historischen Akkumulationsregimen abstrahiert: Agrarwirtschaft bis zum 19. Jahrhundert, gefolgt vom Industriekapitalismus und die

6 Castells führt den Begriff »informational capitalism« ein, um damit das neue technoökonomische Modell vom »industrial capitalism« zu unterscheiden. 
derzeitige Transformation zum Informationskapitalismus. Mit diesen unterschiedlichen Akkumulationsregimen hängt eine grundlegende Transformation der Wertschöpfung zusammen. Im Agrarkapitalismus resultierte der Mehrwert aus der extensiven Nutzung von Land und Arbeitskraft im Produktionsprozeß. Das Neue an der Industrialisierung war die Transformation zur intensiven Nutzung von Technologie und Arbeitskraft, was aber erst durch die fossile Energierevolution möglich gemacht wurde. Nicht nur wurde mit einem geringen Energie-Input eine hohe Energieeffizienz erzeugt, mit diesen Überschüssen wurden auch die sozialen, politischen, kulturellen und ökonomischen Evolutionsprozesse vorangetrieben (Altvater/Mahnkopf 1996). In der gerade ablaufenden Transformation zum Informationskapitalismus ist nicht die Steigerung der Energieeffizienz die Basis für den Mehrwert, sondern die quantitative und qualitative Intensivierung der elektronischen Informations- und Kommunikationsprozesse (Castells 1996).

In der neuen globalen Informationsproduktion liegt, wie Castells betont, die Produktivität in der Wissensproduktion, in der Informationsverfertigung und der symbolischen Kommunikation. Das Besondere ist hier die Anwendung des Wissens auf das Wissen selbst als dem wesentlichen Kern der Produktivität. Es gibt hier einen »virtuous circle« zwischen der Wissenserzeugung, die zu einer verbesserten Informationstechnologie führt, und deren Anwendung zur Verbesserung der Wissensproduktion und Informationsverfertigung (ebd. 1996: 37). Während die Maximierung des Outputs das Ziel des Industriekapitalismus war, verfolgt der Informationskapitalismus das Ziel die Akkumulation von Wissen voranzutreiben und eine höhere Komplexität in der Informationsverfertigung zu erreichen. Diese Wissensproduktion findet aber nicht im abstrakten Raum statt, sie ist eingebettet in die materielle Produktion. Das bedeutet, daß weder die Industrie- noch die Agrarproduktion ihre ökonomische Wichtigkeit - ungeachtet der immer weiter sinkenden Beschäftigungszahlen in diesen Sektoren - in der neuen globalen Ökonomie verlieren. Ganz im Gegenteil, durch die neuen Informationstechnologien wie z.B. die Gentechnologie, eröffnen sich dem Agrarsektor und dem medizinisch/pharmazeutischen Sektor bisher verschlossene Gebiete. Die Erschließung, Manipulierung, Entkodifizierung von menschlichem, tierischem und pflanzlichem Leben und die zukünftige Reprogrammierung des Informationsschlüssels von lebender Materie öffnet - wie auch immer von ethischer Perspektive diese Forschung bewertet wird - neue Tore, die wir im Moment überhaupt noch nicht in ihrem gesamten Zugriff auf Mensch und Natur fassen können (Haraway 1997; Wichterich 1998). Um noch einmal mit Castells zu sprechen:

»Das Besondere der gegenwärtigen technologischen Revolution ist nicht die Zentralität von Wissen und Information, sondern es ist die Anwendung dieses Wissens und dieser Informati- 
on auf die Wissensproduktion und die Vorrichtungen zur Erlangung von Information und der Herstellung von Kommunikation, die auf einem kumulativen Feedback zwischen den Innovationen und den Anwendungen dieser Innovationen beruht.« (Castells 1996: 32).

Die gängige Interpretation der Entwicklung als Abfolge vom Agrar- zum Industriekapitalismus und nun zur Dienstleistungsökonomie ist dagegen eine Simplifizierung dieser äußerst komplexen Verzahnung von Produktionsentwicklungen.

Der technologische Paradigmenwechsel zur Informationstechnologie geht einher mit einem neuen Organisations- und Steuerungsmodus, der aus einer Kombination von Elementen der grundlegenden Ordnungsmuster 'Markt' und 'Hierarchie' besteht: »Einerseits der für Märkte charakteristischen Existenz einer Vielzahl autonomer Akteure, andererseits der für Hierarchien typischen Fähigkeit (oder zumindest Funktion), gewählte Ziele durch koordiniertes Handeln anzusteuern«(Messner 1997: 57). Die Vielfältigkeit von interorganisatorischen Beziehungsgeflechten bezeichnet Manuel Castells (1996: 171) als eine »Netzwerkgesellschaft«, die durch globale strategisch entscheidende ökonomische Aktivitäten entstanden ist. Er definiert Netzwerke als eine spezifische Form von Organisation, die sich durch steigende Autonomie der Teilsysteme bei gleichzeitig wachsender Durchdringung der Teilsysteme sowie durch Überschneidungen mit anderen Netzwerken auszeichnen. Netzwerke sind demnach nicht nur isolierte Phänomene, sondern Ausdruck von qualitativ neuen Organisations- und Steuerungstypen, die die steigende Autonomie zwischen gesellschaftlichen Teilsystemen und der gleichzeitig zunehmenden Interdependenz von Problemlagen zu artikulieren vermögen (Messner 1997: 42; Mayntz 1997). Nicht mehr die vertikale Organisationsform und der entsprechende Steuerungstypus - was von Alfred Chandler als die Form der multinationalen Ära beschrieben wird sondern die »horizontalen Unternehmen « sind heute die Organisationsform der neuen globalen Akteure. Es bestimmen auch nicht mehr die nationalterritorialen Unternehmen das Wachstum der nationalen Ökonomie, denn diese ist zunehmend von Unternehmen abhängig, die auf die globale Konkurrenz und auf globale Interessen reagieren. Die Unternehmen sind Teil eines globalen Netzwerks finanzieller und industrieller Konzerne, die über unterschiedliche, für den kollektiven Output bedeutende Ressourcen verfügen und die nicht länger an die nationalen Volkswirtschaften gebunden sind (vgl. Messner 1997; Die Gruppe von Lissabon 1995).

Die Transformation von vertikalen Strukturen zu horizontalen Netzwerken hat die geschlechtsspezifische fordistische Arbeitsteilung zwischen einerseits hochbezahlten männlichen Fachkräften und weiblichen »Zusatzverdienerinnen« grundsätzlich verändert. Die Globalisierung der Produktion ist mit einer substantiellen Erweiterung der ökonomischen Informalisierung verbunden und basiert auf »Subunternehmen«, deren Arbeitsarrangements 
außerhalb staatlicher Regulation liegen. Harvey (1989) bezeichnete diesen Prozeß als »flexible Akkumulation«. Die exportorientierten transnationalen Konzerne stützen sich auf die billigen, unorganisierten jungen weiblichen Arbeitskräfte, die in den Freihandelszonen die bevorzugten Arbeitskräfte in der Elektronikproduktion, der Bekleidungs- und der Schuhindustrie stellen. Frauen fungieren nicht mehr als Zusatzverdienerinnen zum männlichen Familienernährer, sondern sie subventionieren durch ihre Dauerbeschäftigung vielfach die Saisonarbeit der männlichen Arbeiter oder sind als alleinerziehende Mütter auf Lohnarbeit angewiesen (Ward/ Pyle 1995).

Die Entwicklung der neuen Arbeitsorganisation geht somit einher mit einer neuen Spaltung zwischen hochqualifizierten und nicht ortsgebundenen »Wissensträgern « - meist weiß und männlich - und den gering qualifizierten räumlich gebundenen Arbeitskräften - Frauen aller Hautfarbe sowie jungen, unqualifizierten Männern. Saskia Sassen kritisiert in ihren Studien über die »global cities«, daß sich die Globalisierungsdebatte auf die in der formellen Ökonomie hochbezahlten »Wissensträger« der neuen ortsunabhängigen wissensintensiven Forschungs-, Informations- und Serviceabteilungen konzentriert, während die ortsgebundene Arbeit von unqualifizierten Frauen und Männern im prekären, sozialstaatlich und gewerkschaftlich unabgesicherten »informellen Sektor« kaum berücksichtigt wird (vgl. Sassen in diesem Heft). Durch die einseitige Fokussierung auf nicht ortsgebundene Arbeitsprozesse werden die global agierenen Unternehmen aufgewertet und die lokale Arbeit erfährt gleichzeitig eine Abwertung. Diese »unsichtbaren« Arbeiterinnen sind aber die »Träger« der ortsgebundenen materiellen Produktionsprozesse, die die datentechnisch ortsunabhängige Informationsindustrie erst möglich machen. Wenn man die prekären Tätigkeiten von Frauen und Immigranten als einen Teil des globalen Prozesses versteht, der die globale Produktion auch immer wieder lokalisiert, dann sind Frauen und Immigranten neben der Internationalisierung von Kapital ein wichtiger Teil des Globalisierungsprozesses. Die Kehrseite der »wissensgestützten entterritorialisierten Dienstleistungsgesellschaft« ist somit die Ausdehnung des »informellen Sektors ${ }^{7}$ mit einer starken Zunahme von prekären, ungeschützten und risikoreichen Beschäftigungsformen (Mahnkopf 1997; Ward/Pyle 1995; Ward 1990; Rowbotham/Mitter 1994).

Der informelle Sektor beschäftigt weltweit weit mehr Frauen als der formelle Sektor. ${ }^{8}$ Die tragenden Säulen des informellen Sektors sind Subunter-

7 Der informelle Sektor umfaßt ein breites Band von legalen bis hin zu illegalen Tätigkeiten, wie die neue »Heimarbeit«, Arbeit in den neugegründeten Freien Produktionszonen, in Sweatshops oder Arbeit als Straßenverkäufer, Dienstboten, illegale Arbeit in der Landwirtschaft, illegale Prostitution etc. - alles Tätigkeiten, die sozialstaatlich und tarifvertraglich nicht geregelt sind (Mahnkopf 1997; Ward/Pyle 1995).

8 Diese Information erhielt ich in einem Interview mit Dr. Nilufer Cagatay, Ökonomin im 
nehmen, die für die großen und meist transnationalen Konzerne der formellen Ökonomie produzieren. Besonders in den Freien Produktionszonen, die in Mittelamerika, Teilen von Afrika und Ost-Asien zu finden sind, hat die Dezentralisierungsstrategie transnationaler Unternehmen die neuen exportorientierten »Weltmarktfabriken « hervorgerufen. Vor allem junge und verheiratete Frauen sind gefragt, die unter dem Minimallohn arbeiten und sich nur selten gewerkschaftlich organisieren. An der Grenze zwischen den USA und Mexiko arbeiten diese »Weltmarktfabriken « auf Vertragsbasis für amerikanische transnationale Konzerne wie J.C. Penney, Sears \& Roebuck, Walmart und Montgomery Ward. Die »maquila businesses« profitieren von der hohen Arbeitslosigkeit und der Bereitschaft der Frauen und Männer zu niedrigen Löhnen ihre Existenz zu sichern. ${ }^{9}$ Diese Produktionszonen genießen einen extraterritorialen Status: Importe wie Exporte spielen sich innerhalb der Freien Produktionszonen ab, die vom nationalen Territorium abgeschnitten sind; sie bieten keinen Wohnraum für die ArbeiterInnen; Gebäude und Einrichtungen sind von Steuern befreit, die Konzerne entrichten weder Gemeindeabgaben noch Boden- oder Einkommenssteuer, zudem sind Importe wie Exporte vom Zoll befreit; die Aufnahmeländer stellen die Infrastruktur, bauen Straßen und Hafenanlagen, und bieten zu günstigen Preisen Telefon, Wasser und Energie (Lemoine 1998).

Die Produktion in transnationalen Konzernen ist zunehmend verbunden mit lokalen informellen exportorientierten Tätigkeiten und den ausgelagerten Heim- und Hinterhoftätigkeiten. Transnationale Unternehmen in Indonesien, Taiwan und Südkorea verlagern Teile der Produktion zunehmend in ländliche Gebiete. Die Konzerne verschaffen sich durch die noch weitgehend bestehenden Haushaltsökonomien einen Vorteil, da sie Frauen unter dem Subsistenzminimum entlohnen können. Somit entlastet die Subsistenzarbeit der Frauen im Haushalt die Kosten der transnationalen Konzerne. Die Suche vieler dieser Unternehmen nach immer neuen Billiglohnländern bedeutet andererseits, daß Firmen in kürzester Zeit entweder die Produktion in Hinterland-, Hinterhof-, und Straßenrandökonomien auslagern oder automatisieren (Ward/Pyle 1995).

Im Unterschied zur fordistischen Produktion handelt es sich bei den neuen »Weltmarktfabriken« nicht um große Werkshallen mit einer zentralisierten Massenproduktion. Diese Zulieferbetriebe sind netzwerkförmig auf dem Globus verteilt. Die in Hinterland-, Hinterhof- und Straßenrandökonomien konzentrierten, niedrigbezahlten und arbeitsintensiven Tätigkeiten erscheinen nicht nur in Entwicklungsländern. Auch in Mittel- und Osteu-

United Nations Planning Department (UNDP), in New York am 11. März 1998.

9 Frauen stellen in den »maquila business « überall die Mehrheit: 58\% in Mexiko (1995), 60 bis $62 \%$ in Costa Rica, 70 bis $75 \%$ in Honduras, $78 \%$ in Guatemala (1993) und $60 \%$ in der Dominikanischen Republik (1992) (Lemoine 1998). 
ropa sowie in den westlichen Industrieländer hat das Zusammenwirken von Globalisierung, Informalisierung und Transnationalisierung zu zunehmender Flexibilisierung der Beschäftigungsformen und $\mathrm{zu}$ einer geschlechtsspezifischen Verfestigung von Niedriglohnarbeit geführt (Wichterich 1998; Mahnkopf 1997). Transnationale Konzerne sind in der Semiperipherie von Europa wie z.B. Griechenland, Spanien und Irland genauso zu finden wie in den Vereinigten Staaten. Viele der europäischen Textil- und Kleidungsindustrie, wie der italienische Erzeuger Benetton, sind Marketing-Unternehmen, die die Subunternehmen, die auf häuslicher (weiblicher) Produktion und auf »sweatshops« beruhen, global koordinieren (Harvey 1989). In den USA findet man die transnationalen Konzerne in denselben Industrien (z.B. Elektronik- und Bekleidungsindustrie) wie in den Billiglohnländern der sogenannten dritten Welt. Im Gegensatz zu der weitverbreiteten Annahme, daß die niedrigqualifizierten und arbeitsintensiven Industriesektoren in Billiglohnländer abgewandert sind, zeigt das US-amerikanische Beispiel gerade das Gegenteil. Die Konsumelektronik- und die Textilindustrie sind keine schrumpfenden Industriezweige. Allein die Zahl der Beschäftigten in der Bekleidungsindustrie ist größer als diejenige in der Auto-, Stahl-, und Elektronikindustrie zusammen (Fernández Kelly 1989).

Trotz gravierender Differenzen gibt es Gemeinsamkeiten zwischen den osteuropäischen Transformationsgesellschaften, Teilen der in die Weltwirtschaft (partiell) integrierten »Dritten Welt« und den Industrieländern: Es entstehen überall »Niedriglohnzonen« (Mahnkopf 1997). Diese »Enklaven der Informalität« sind zum dauerhaften Bestandteil der formellen Ökonomie geworden. Somit ist die Informalisierung von Beschäftigungsverhältnissen weder eine Randerscheinung noch ein Übergangsphänomen. Sie ist ein immanenter Teil der ökonomischen Globalisierung. Zunehmend entstehen im Norden neue Süden, wie die Gruppe von Lissabon (1995: 79) bemerkt, und im Süden entstehen neue Norden. In diesen prekären und flexiblen Arbeitsstrukturen entwickeln sich neue geschlechtsspezifische und ethnische Hierarchien. Zum Beispiel sind in der Textilindustrie von Miami die Hersteller vorwiegend jüdische Männer, die »subcontractors« bestehen zu 90\% aus kubanischen Männern und in den sweatshops findet man zu 95\% kubanische Frauen (Fernández Kelly/Garcia 1992). Im informellen Sektor entstehen somit neue Geschlechterdifferenzen und -hierarchien, die vor allem durch klassenspezifische sowie ethnische und nationale Zugehörigkeiten noch einmal gravierend verschärft werden. Männer kontrollieren weitgehend den unregulierten Bereich des subcontracting, während zunehmend mehr Frauen (vor allem Immigrantinnen) als Arbeiterinnen in sweatshops zu finden sind (Fernández Kelly/Sassen 1995). 


\section{Die neuen Genderregime und -ordnungen}

Was kann nun als vorläufige Bilanz in Bezug auf geschlechtsspezifisch segregierte Arbeitsmärkte und -kulturen und geschlechtsspezifische Formen der Macht und des »empowerment« in den neuen Genderregimen ausgesagt werden? Hier wird davon ausgegangen, daß sich die drei Säulen der fordistischen Genderregime und -ordnungen: 1) das männliche Familienernährermodell; 2) daß Frauen mit dem Reproduktionsbereich in der privaten Sphäre assoziert werden; 3) die Trennung von geschlechtsspezifischer Privatheit und Öffentlichkeit, durch die Globalisierung einer grundlegenden Veränderung unterworfen sind. Die Neu-Konfiguration, die sich erst in schwachen Konturen ausmachen läßt, findet auf vielen Ebenen statt. Erstens ist das fordistische Modell des männlichen Familienernährers zunehmend ein Phänomen der Vergangenheit. Zweitens ist die fordistische geschlechtsspezifische Trennung von Privatheit und Öffentlichkeit und der mit ihr assoziierten Teilung in Produktions- und Reproduktionsarbeit nicht mehr haltbar. Drittens zeigt sich bei zunehmender Gleichheit zwischen Frauen und Männern der Mittelschicht eine zunehmende Ungleichheit und Ausdifferenzierung zwischen Frauen nach schichtspezifischer, ethnischer und nationaler Zugehörigkeit (Friese 1995). Viertens entsteht eine geschlechtsspezifische Gesellschaftspaltung zwischen einerseits der meist männlichen entterritorialisierten Geldgesellschaft und der an den Nationalstaat geknüpften (meist weiblichen) Arbeitsgesellschaft. Diese Veränderungen sind nicht nur einseitig negativ zu deuten. Sie führen auch zur Schwächung und Auflösung von lokalen, patriarchal geprägten Kulturen und Herrschaftssystemen.

a) Die Unterminierung des fordistischen Familienernährermodells

Durch die Globalisierung erodierte zu einem erheblichen Teil das materielle Fundament, auf dem das Konstrukt des männlichen Ernährers und seiner abhängigen Frau entstanden ist. Die seit den 70er Jahren steigende Anzahl der Doppelverdiener ist ein Nebenprodukt dieser Entwicklung. Doppelverdienerhaushalte können in zwei Gruppen aufgeteilt werden: Einerseits finden sich die verhältnismäßig wohlhabenden »professionals«, die in der formellen Ökonomie eingebettet sind. Eine größere Gruppe befindet sich auf der mittleren und unteren Ebene der Ökonomie, die auf den »Zusatzlohn « von Frauen angewiesen ist, um einen gewissen Lebensstandard entweder zu halten oder zu verbessern. Außerdem hat die Zahl der Alleinerziehenden (meist weiblich) drastisch zugenommen. Im Jahre 1988 waren in den USA $67 \%$ der alleinstehenden Mütter, 53\% von Müttern mit Kindern unter drei Jahren in Lohnarbeit zu finden und von den Doppelverdienerhaushalten hatten 53\% Kinder (Fernández Kelly/Sassen 1995; Ward/Pyle 
1995). Der Unterschied zur fordistischen männlichen Beschäftigungsnorm ist, daß die derzeitige Informalisierung der Beschäftigung, die durch die »Feminisierung der Beschäftigung « gekennzeichnet ist, den Anspruch auf einen Familienlohn nicht mehr erfüllt und daher keine ökonomische Sicherheit gewährleisten kann.

Die zunehmende Integration von Frauen in den Arbeitsmarkt hat zu neuen Genderdefinitionen und Werteverschiebungen in den Genderrollen beigetragen. Die fordistische Wertvorstellung von der Frau als vom männlichen Ernährer abhängig wird durch die zunehmende Individualisierung der Frau unterminiert. Frauen aus Mexiko, die in den USA leben, schildern diese neue Wertewanderung folgendermaßen:

»Früher, wenn eine Frau außerhalb des Haushalts arbeitete, wußten alle, daß dies getan wurde, um dem Mann zu helfen; aber es war seine Pflicht die Familie zu ernähren. Jetzt ist es unsere Pflicht; von Frauen wird erwartet, außerhalb des Haushalts zu arbeiten, ob sie es wollen oder nicht« (Fernández Kelly/Sassen 1995: 112/113).

b) Die Neudefinition von Privatheit/Öffentlichkeit und von Produktion/Reproduktion

Die Trennung von Produktion/Reproduktion und Privatheit/Öffentlichkeit, die zentral für das mit dem Fordismus einhergehende Genderregime war, wird durch die Globalisierung weitgehend aufgehoben. Erstens spielen sich die Prozesse der Produktion und Reproduktion (auch der sozialen Reproduktion) zunehmend in einem breiten Band von informellen, formellen und Haushaltsökonomien ab. ${ }^{10}$ Die begriffliche Unterscheidung zwischen Privatheit und Öffentlichkeit berücksichtigt nicht, daß die tägliche Arbeit von vielen Frauen in einer »triple shift « (Hossfeld 1990) zwischen formeller, informeller und Familien- oder Subsistenzarbeit stattfindet. ${ }^{11} \mathrm{Ob}$ diese »triple-shift« von Frauen in der Karibik, in Asien oder in Silicon Valley verrichtet wird, sie hat gemein, daß Frauenarbeit eine Kombination von Beschäftigung in der formellen transnationalen Produktion, in informellen Bereichen und in der Subsistenzwirtschaft darstellt. Die Grenzen dieser »triple shift« sind äußerst offen für Frauen und relativ rigide für Männer. Um ihr ökonomisches Überleben zu sichern, verbringen Frauen bis zu 16 Tagesstunden in dieser »triple-shift «. Männer sind im Vergleich dazu kaum in die Haushaltsökonomie involviert und arbeiten entweder in der formellen oder in den informellen Ökonomie (Ward/Pyle 1995).

10 Diese Beschäftigungsveränderungen sind tendenziell noch immer auf die Schwellen- und Transformationsländer begrenzt, aber diese Form verbreitet sich - wie das USamerikanische Beispiel zeigt - auch zunehmend in den westlichen Industrieländern.

11 Formelle Arbeit bezieht sich auf geschützte und regulierte Lohnarbeit, informelle auf flexible und ungeschützte Arbeit und Familienarbeit ist weder reguliert noch monetär vergütet (Ward/Pyle 1995). 
Bereits in den 70er Jahren wiesen Feministinnen darauf hin, daß die Gegenüberstellung von Markt einerseits und Familie andererseits und die geschlechtsspezifische Besetzung dieser Sphären eine Eindeutigkeit ausdrückt, die in sich durchaus widersprüchlich ist. Regina Becker-Schmidt meinte,

»Was uns gemeinhin als öffentlich gilt, z.B. die soziale Marktwirtschaft, hat als kapitalistische sehr wohl privateigentümlichen Charakter, und umgekehrt das scheinbar Allerprivateste, die Familie, erfüllt durchaus Aufgaben im Dienste der Öffentlichkeit - Erziehung, Regeneration von Arbeitskraft, Altenversorgung.«(Becker-Schmidt 1992: 219)

Die feministische Erweiterung des Begriffs »Arbeit« auf monetär nicht vergütete Arbeiten im Reproduktionsbereich bleibt m.E. der konventionellen Konzeption von zwei komplementären Sphären von Produktion und Reproduktion verhaftet. In dem Maße, wie die männliche Ernährerrolle immer mehr in den Hintergrund tritt, werden Frauen zunehmend gezwungen ihre Existenz in einer Mischung von privatem und öffentlichem Bereich zu sichern. Sie verrichten auf Abruf bezahlte Tele- und Heimarbeit in der privaten Sphäre (Haushalt), oder sie sind in sweatshops (weder privat noch öffentlich reguliert) zu finden und haben vielfach ihre Babies auf den Rücken geschnallt. ${ }^{12}$ Dies bedeutet, unter anderem, daß der Begriff einer regulären, statistisch abgrenzbaren Arbeitszeit, den man aus der formellen Ökonomie kennt, in den Transformations- und in den Entwicklungsländern nicht mehr greift, um die neuen Beschäftigungsformen einzuordnen. ${ }^{13} \mathrm{Zu}-$ nehmend ist Frauenarbeit eine Kombination von bezahlter Beschäftigung in transnationalen Konzernen, im informellen exportorientierten Bereich und unbezahlter Haus- und Subsistenzarbeit. Joan Smith und Immanuel Wallerstein haben bereits in den 70er Jahren eine Rekonzeptualisierung der Sphären von Familie, Arbeitsplatz, und Staat vorgeschlagen. Anstatt des Begriffs »Familie« und der Trennung zwischen monetärer Produktion und nicht monetärer Reproduktion, benutzen sie den Begriff »Haushalt«. »Haushalte« sind demnach Gruppen von Familienmitgliedern, Mitbewohnern oder auch Nachbargemeinschaften, die ihre Einkommen und Ressourcen bündeln. Mit diesem Begriff wird zwischen monetärer und nichtmonetärer Arbeit und zwischen Familienmitgliedern, Freunden und Nachbarn nicht mehr differenziert. Der Begriff »Einkommen« wird auf nichtmonetäre Bereiche wie die Subsistenzproduktion und die Nachbarschaftshilfe ausgedehnt (Smith/Wallerstein 1992: 6-9).

In diesen neuen Beschäftigungsformen, die besonders in den Billiglohnländern zu erkennen sind, wird auch die Genderidentität neu konstruiert. Während im fordistischen Modell die Frau dem Reproduktionsbereich zugerechnet wurde (Frau \& Kinder), spielt in der globalen Ökonomie nur mehr

12 Siehe das Titelbild von K. Ward Women Workers and Global Restructuring (1990).

13 Diese unregulären Beschäftigungsformen gehören zunehmend zum US-amerikanischen Alltag. 
die »Frau« als Individuum eine Rolle. Das ökonomische Interesse im informellen Sektor bezieht sich auf »ihre« Arbeitskraft, und die Vereinbarkeit von Reproduktion und Produktion wird gänzlich in die Privatsphäre abgeschoben. Nicht nur wird von einem ökonomischen Standpunkt die Reproduktion »unsichtbar«, mit der Informalisierung der Ökonomie wird auch, daß jeglicher Anspruch auf Kinderversorgung oder andere soziale Leistungen nicht mehr anerkannt. Ob Frau durch kranke Kinder an der Arbeit in den Freihandelszonen verhindert wird, ist nicht einmal mehr Gegenstand einer öffentlichen Diskussion. Ihr Platz wird von anderen eingenommen (Lemoine 1998). Frau ist durch die Abstraktion von ihren Reproduktionsleistungen in der globalen Ökonomie nun dem männlichen Individuum als verfügbare Arbeitskraft »gleichgesetzt«. Die weibliche Reproduktionsarbeit, die im fordistischen Modell trotz ihrer »Verbannung « in die private Sphäre gesellschaftlich - jedoch nicht monetär - anerkannt wurde, wird durch die derzeitige Informalisierung »ausgeblendet $«$. Somit wird der Bereich der Reproduktion als eine ökonomische »Externalität « definiert und das dialektische Verhältnis zwischen Markt und nicht-marktförmigen Aktivitäten verschwindet aus dem neoliberalen Diskurs (vgl. Elson 1994; Mann 1994).

c) Die zunehmende Ungleichheit zwischen Frauen

Die steigende Integration der Frauen in den Arbeitsmarkt führt zu immer größeren Differenzierungen zwischen Frauen unterschiedlicher Ethnien, Schichtzugehörigkeit und Nationalitäten. Obwohl die Mitglieder der reichen »Clubgesellschaft« meistens die »new boys « sind, wie Wendy Larner (1996) die neuen »Macher« des neoliberalen Neuseeland-Modells bezeichnet, sind gut ausgebildete Mittelschichtsfrauen in den mittleren wissensund informationsintensiven Wirtschaftssektoren keine Seltenheit mehr. Linda McDowell (1997) porträtiert die durchaus vorsichtige, aber doch zunehmende Öffnung der Londoner »City«, bisher eine männliche Finanzhochburg, für die neuen weiblichen »professionals «. Durch den expandierenden internationalen Dienstleistungssektor ist es jungen hochgebildeten und weiblichen »professionals « durchaus - trotz aller Widersprüchlichkeit - gelungen, in den oberen Etagen der Finanz- und Geschäftswelt in den Global Cities Einzug zu halten.

Diese Arbeitsmarktsegmentierung in eine hochbezahlte »postmoderne Informationsökonomie « einerseits und den expandierenden informellen Sektor der »laboring poor« andererseits führt zu immer mehr Ungleichheiten zwischen Frauen. Die niedrigqualifizierten Dienstleistungstätigkeiten sind nicht nur ein wichtiger Bestandteil der Infrastruktur der formellen Ökonomie. Sie ermöglichen vor allem sozial privilegierten Frauen in Europa, Nordamerika und anderswo den Einstieg in eine berufliche Karriere. Solange Frauen, unabhängig von ihrer sozialen Schichtzugehörigkeit für die 
Reproduktionsarbeiten weiterhin verantwortlich bleiben, sind die Rahmenbedingungen zum Einstieg in die »männliche « Berufswelt geschlechtsspezifisch bestimmt. Weiße sozial privilegierte Frauen können aber immerhin auf billige, meist zugewanderte - teils illegale - Migrantinnen für die Reproduktionsarbeit zurückgreifen. Ohne diese aus den Entwicklungs- und Transformationsländern zugewanderten Frauen und ohne adäquate staatliche Kinderversorgung ist den hochqualifizierten Frauen mit Kindern der Einstieg in Positionen verwehrt, die Mobilität erfordern und wenig familienfreundliche Arbeitszeiten voraussetzen. Ob diese Tätigkeiten von (meist überqualifizierten) Polinnen in Deutschland oder von schwarzen Frauen und lateinamerikanischen Immigrantinnen in den USA verrichtet werden, sie führen zu einer neuen internationalen Arbeitsteilung zwischen der »Herrin« einerseits und der meist aus einer anderen Ethnie und Klasse stammenden »Dienstbotin « andererseits. Somit ist eine berufliche Frauenkarriere der europäischen und nordamerikanischen Mittel- oder Oberschichten nur in den Grenzen von Ethnizität, Klasse und Geschlecht zu realisieren (vgl. Friese 1995; Mahnkopf 1997).

d) Geschlechtsspezifische Gesellschaftsspaltungen

Globalisierung führt zu Gesellschaftsspaltungen zwischen der Arbeitsgesellschaft, die bislang unauflöslich an den Nationalstaat geknüpft ist, und der Geldgesellschaft, die mit der Deregulierung der Finanzmärkte nicht mehr an den Nationalstaat gebunden ist. Dieser Gegensatz zwischen einer globalen Geldgesellschaft einerseits und einer an den Nationalstaat geknüpften Arbeitsgesellschaft andererseits ist durch die Herauslösung (Entbettung) der monetären Sphäre aus realen ökonomischen Prozessen entstanden (Altvater/Mahnkopf 1996). Der durch die Globalisierung eingeleitete Gegensatz zwischen Geldgesellschaft und Arbeitsgesellschaft erweist sich als einer der wichtigsten Gründe für die Krise des Wohlfahrtsstaats. Die Mehrzahl der Frauen und Männer bleibt aber auf die nationale »Solidaritäts- und Schicksalsgemeinschaft « für ihre soziale Sicherung angewiesen. Es war ein Verdienst des Sozialstaates, die arbeitenden Klassen als politische Staatsbürger in den Staat zu integrieren. Der Nationalstaat steckte somit den Horizont der Arbeitsgesellschaft ab. Innerhalb der Grenzen des nationalstaatlichen Raumes wurde die keynesianische, geschlechtsspezifische Vollbeschäftigungsgarantie zum ökonomischen Stabilitätsfaktor und zum Vehikel der Identitätsbildung der BürgerInnen, auch wenn diese bürgerlichen Rechte, wie Carole Pateman (1994) betont, politische Rechte sind, die eine spezifisch moderne Form von Patriarchat etabliert hat.

Die Mitglieder der Geldgesellschaft, zumeist weiß und männlich, sind aber nicht mehr auf den territorial begrenzten Raum angewiesen. Die Subjekte der Geldgesellschaft, so Altvater und Mahnkopf, sind Mitglieder einer glo- 
balen »Clubgesellschaft«, wo nur die Codes des Mediums Geld verstanden werden. Sie sind nicht mehr auf den Wahlzettel der Stimmbürger angewiesen und verwenden ihn selbst nur, um jegliche Steuererhöhung oder soziale Umverteilung an der Wahlurne zu stoppen. Die Geldvermögensbesitzer gehören zwar pro forma einer Nationalität an, sie sind aber nicht mehr gebunden an die national-begrenzte »Schicksals- und Solidaritätsgemeinschaft «. Die Gestalt der freien StaatsbürgerInnen bleibt formal erhalten, aber die sozialen Rechte und die ökonomische Basis für die politische Gleichheit werden mit der Globalisierung tendenziell zurückgenommen. Die Ansprüche der Arbeitsgesellschaft auf gesellschaftliche Umverteilung werden unter den Bedingungen des weltweiten »single price« ausgehöhlt. Somit kollidieren die »Sachzwänge« der nationalen Wettbewerbsfähigkeit mit der Logik des sozialen Konsens.

Frauen sind aber durch ihre dreifache Verknüpfung mit dem Sozialstaat als Angestellte, Klientinnen und Konsumenten - noch einmal besonders von der Sozialkrise betroffen. Sie werden durch die öffentlichen Sparmaßnahmen in zweifacher Weise in Mitleidenschaft gezogen: Es verringern sich nicht nur die staatlich unterstützten Betreuungsangebote für Kinder. Im Alten-, Pflege-, Gesundheits- und Bildungsbereich zu kürzen heißt, diese Arbeiten wieder unbezahlt von Frauen haushalts- und familienintern bewältigen zu lassen. Diese sich gegenwärtig vollziehende Privatisierung von öffentlichen Dienstleistungen zerstört die notwendigen Voraussetzungen für die Berufstätigkeit insbesondere von weniger qualifizierten Frauen, gleichzeitig entfallen die staatlichen »Frauen«-Arbeitsplätze, die im Keynesianischen Wohlfahrtsstaat eine wichtige Integrationsrolle für die Erwerbsbeteiligung von Frauen hatten (Jensen/Hagen/Reddy 1988).

Der gegenwärtige neoliberale Diskurs, der auf die Reprivatisierung der sozialen Leistungen abzielt, impliziert, daß die soziale Reproduktion in steigendem Maße wieder in die private Sphäre verlagert werden soll. Dabei spielt die traditionelle hetero-patriarchale Familie eine zentrale Rolle. Die Pflegeleistungen familienintern wieder bewältigen zu lassen, bedeutet aber nicht, daß diese Arbeiten auf »alle« Familienmitglieder gleich verteilt werden. Nicht der Familie wird diese Verantwortung aufgebürdet sondern der Frau. Die Forderung soziale Leistungen zu re-privatisieren setzt aber gewisse Genderregime voraus, die heute immer mehr in Frage gestellt werden. Die ökonomischen und strukturellen Bedingungen, die das fordistische Genderregime untermauert haben, sind größtenteils nicht mehr vorhanden. Zur heutigen Realität gehört, daß Frauen in absehbarer Zeit nicht mehr in die Privatsphäre zurückkehren können oder wollen. Wie die Reproduktionsfrage in diesen durch die Globalisierung ausgelösten Veränderungen der Genderregime gesellschaftlich gelöst werden wird oder soll, ist derzeit eine offene Frage. 
Durch die globale Konkurrenz wird, wie bereits erwähnt, der Staat immer mehr zum Wettbewerbsstaat. Dies bedeutet, daß im Staat einzelne Institutionen oder Abteilungen aufgewertet und andere abgewertet werden. Im allgemeinen findet eine Aufwertung der ökonomischen gegenüber den politischen Interessen des Staates statt. Dies impliziert aber nicht, wie vielfach angenommen wird, daß die Globalisierung den Nationalstaat einfach nur schwächt, aushöhlt oder marginalisiert. Die einseitige Fokussierung auf den Abbau des Sozialstaats als Folge der Globalisierung versperrt m.E. den Blick auf die Verlagerung und Entstehung von neuen Machtzentren, die eng mit den Finanz- und Wirtschaftsabteilungen innerhalb des Staates, verbunden sind. Diese Prozesse beinhalten auch neue Konflikte innerhalb des Staatsapparates. Die mit dem Sozialstaat verbundenen institutionalisierten Mehrheiten haben ihre »Macht« gegenüber den ökonomischen und den Finanzinteressen verloren. Analysiert man den Zugang zum Staat als eine »strategische Selektivität« (Jessop 1990) ${ }^{14}$, dann wird durchaus verständlich, warum die mit dem Sozialstaat verbundenen gesellschaftlichen Kräfte in der Globalisierung erstmals ihren Zugang zur staatlichen Machtzentrale größtenteils eingebüßt haben.

\section{Fazit}

Feministische Wissenschaftlerinnen stehen nun vor neuen Herausforderungen. Es stellt sich nicht »nur « die Frage, ob der Nationalstaat in seiner Struktur vergeschlechtlicht ist oder ob der Staat in einer vergeschlechtlichten Gesellschaft existiert, Feministinnen stehen vor der Aufgabe, die strukturellen und strategischen Ungleichheiten in den neuen horizontalen Netzwerkstrukturen aufzuspüren. Die globalen Akteure sind heute transnationale Firmen, Banken, Versicherungs-, Informations- und Ratingagenturen; internationale Institutionen wie z.B. die Welthandelsorganisation, die Weltbank, der Internationale Währungsfonds; nicht-staatliche Organisationen wie z.B. Amnesty International, die Olympische Sportorganisation oder transnationale Verbände von Ärzten und Ökonomen und schließlich auch die Mafia und internationale Finanzspekulanten (Strange 1996). Die expandierende Zunahme und Verflechtung zwischen staatlichen und nichtstaatlichen Akteuren kann mit den Begriffen »vergesellschafteter Staat « oder »verstaatlichte Gesellschaft « nicht mehr angemessen beschrieben werden (vgl. Messner 1997). Die neuen Netzwerkstrukturen signalisieren eine Transformation der »institutionellen Hülle des Nationalstaates« inner-

14 Das Konzept einer »strategischen Selektivität« basiert auf der Annahme, daß ein bestimmter Staat oder ein bestimmtes politisches Regime spezifischen Interessengruppen eher zugänglich ist als anderen, sowohl hinsichtlich politischer Strategien als auch der Interessenvertretung (Jessop 1990). 
halb eines national begrenzten Territoriums. Nicht territoriale Eroberungen erzwingen eine Machtverschiebung auf staatlicher Ebene, wie dies im imperialen Zeitalter der Fall war, es entsteht vielmehr eine neue transnationale „Geographie der Macht« (Sassen 1996), deren Ausgangspunkt die Grenzen von Staat und Markt sind. Für die feministische Forschung bedeutet dies, die institutionelle Materialität des Staates in diesen Netzwerkstrukturen zu analysieren und zu untersuchen, wie diese Materialität transformiert und reorganisiert wird. Feministinnen sind somit gefordert, »global zu denken und lokal zu handeln.«

Die Globalisierung darf aber nicht nur negativ für die existierenden Genderverhältnisse betrachtet werden. Es ist unumstritten, daß die Informalisierung der Ökonomie die Arbeitsmarktintegration von Frauen drastisch erhöht hat. Studien zeigen, daß die globalen exportorientierten »Weltmarktfabriken « eindeutig Arbeiterinnen präferieren. Diese »Feminisierung der Beschäftigung « geht deutlich mit der Verbreitung flexibler Arbeitsstrukturen einher, welche die lebenslange, existenzsichernde fordistische (männliche) Vollbeschäftigung zunehmend verdrängen. Aber trotz niedriger Löhne bieten transnationale Konzerne im Vergleich zur heimischen Industrie bessere Arbeitsbedingungen und höhere Löhne (Lemoine 1998; Ward/Pyle 1995). ${ }^{15}$ Für weibliche Arbeitskräfte ist die Beschäftigung in diesen »Weltmarktfabriken« zwar oft die einzige Möglichkeit einer Erwerbsarbeit, aber sie kann zu mehr Unabhängigkeit von ihren Familien und Männern führen. Außerdem zeigen neuere Studien, daß Frauen keineswegs nur passiv ihre Unterdrückung dulden. Es sind zunehmend Frauen, die zu den aktiven Gewerkschaftsmitgliedern in Süd-Korea, den Philippinen und Südafrika gehören und gegen Dequalifizierung, Lohndrückerei und anderen Taktiken der transnationalen Konzerne kämpfen (Ward/Pyle 1995). Somit ist das Bild der »passiven « Arbeiterin nicht nur eine ideologische Karikatur; es zeigt vor allem die widersprüchlichen Prozesse der Globalisierung. Frauen werden von transnationalen Firmen in den Weltmarktfabriken bevorzugt eingesetzt, weil sie als passiv und gewerkschaftlich schwer organisierbar eingestuft werden. Aber gerade die Arbeitsmarkterfahrung zwingt Frauen erste Ansätze des Widerstands zu formulieren, die auf die neue Situation zugeschnitten sind. Diese Arbeitskämpfe verändern die Situation auf der lokalen Ebene jedoch oft nur kurzfristig und bewirken keine strukturellen Veränderungen in den transnationalen Strategien (Ward/Pyle 1995).

Es ist auch nicht zu leugnen, daß die Ausbeutung auf dem globalen Markt von einer Auflösung und Schwächung der lokalen, patriarchal geprägten Kulturen begleitet ist, was für die Frauen durchaus positiv ist: Sie können

15 In Honduras beträgt der Mindestlohn in den maquilas im Schnitt 40 Lempiras pro Tag (5,60 DM), gegenüber 30 Lempiras in der restlichen Industrie (Lemoine 1998). 
sich der männlichen Unterwerfung durch ihre Einbindung in den globalen Markt entziehen. Andererseits verlieren Frauen aber auch den Schutz, den die lokalen patriarchalen Kulturen bieten. Dieser Verlust von Frauenrechten auf nationalstaatlicher Ebene wird aber durch internationale Konventionen wie z.B. der Vierten Weltkonferenz in Beijing von 1995 und der offiziellen $»$ Beijing Declaration ${ }^{16}$ - wenn auch nur marginal - relativiert. Wir sind Zeugen, so Saskia Sassen (1996), einer zunehmenden Verlegung von wichtigen Aspekten der staatlichen Souveränität auf supranationale, nichtstaatliche oder private Institutionen. Dies bringt eine potentielle Stärkung alternativer Subjekte des internationalen Völkerrechts mit sich wie z.B. der NGOs und der Minoritäten in internationalen Foren. Diese neuen Organisationsstrukturen jenseits des Staates haben auch Implikationen für die Konzeption von »Bürgerschaft«. Beides könnte dazu beitragen, Frauen - entweder als Individuen oder als Kollektiv - innerhalb des Völkerrechts als Subjekte anzuerkennen. Menschenrechtsorganisationen sowie Feministinnen fordern seit geraumer Zeit, die anarchistische Konzeption der internationalen Staatensysteme aufzuheben und internationale Gremien aufzufordern, innenpolitisch zu intervenieren, wenn die Gefahr der Menschenrechtsverletzung besteht. Mit dem Slogan, »Frauenrechte sind auch Menschenrechte « haben Frauen einen völkerrechtlich einklagbaren Grundsatz gefordert. Skeptikern, die diese Forderung als einen »Tiger ohne Krallen« betrachten, sollten an die Helsinki-Beschlüsse von 1975 erinnert werden, die durch die »Charta 77« einen großen Impuls auf die Oppositionsbewegungen in Osteuropa hatten.

Globalisierung ist nicht nur ein Schreckensszenario. Es ist m.E. weder theoretisch hilfreich, noch macht es handlungsfähig, wenn Frauen in den $\mathrm{Zu}-$ stand eines erstarrten Kaninchens geraten, sobald sie mit der Schlange Globalisierung konfrontiert werden. Es ist nicht zu leugnen, daß die voranschreitende Globalisierung den Spielraum nationaler Staaten und politischer AkteurInnen verengt hat. Es muß aber auch daran erinnert werden, daß die Auflösung der nationalstaatlich begrenzten fordistischen Genderregime und -ordnungen durchaus eine Chance bietet, neue feministische Konzepte und Strategien zu entwickeln, die Frauen global endlich zu gleichberechtigten Bürgerinnen werden läßt.

\section{Literatur}

Aglietta, M., 1979: A Theory of Capitalist Regulation, London.

Altvater, E., 1997: Markt und Demokratie in Zeiten von Globalisierung und ökologischer Krise, in: Altvater, E., Brunnengräber, A., Haake, M., Walk, H., Vernetzt und verstrickt. Münster, S. 241-256.

16 Siehe Platform for Action and the Beijing Declaration, Fourth World Conference on Women, Beijing, China, 4-15 September, 1995. 
Altvater, E., Mahnkopf B., 1996: Grenzen der Globalisierung, Münster.

Bauman, Z., 1996: Glokalisierung oder: Was für die einen Globalisierung, ist für die anderen Lokalisierung, in: Das Argument, S. 653-664.

Beck, U., 1997: Heraus aus dem nationalen Politikbiotop, in: Die Tageszeitung. 13. Juni.

Becker-Schmidt, R., 1993: Geschlechterdifferenz - Geschlechterverhältnis: soziale Dimensionen des Begriffs 'Geschlecht', in: Zeitschrift für Frauenforschung, Nr. 1 / 2 , S. 37-46.

Bourdieu, P., 1998: Die Sachzwänge des Neoliberalismus, in: Le Monde Diplomatique, März.

Castells, M., 1996: The Rise of the Network Society: The Information Age: Economy, Society and Culture, Volume I, Oxford.

Chandler, A., 1977: The Visible Hand: The Managerial Revolution in American Business. Cambridge.

Connell, R.W., 1987: Gender and Power. Society, the Person and Sexual Politics.Stanford.

Eisenstein, Hester, 1990: Femocrats, Official Feminism and the Uses of Power, in: Sophie Watson (Hrsg)., Playing the State. Austrialian Feminist Intervention. London.

Demirovic, A., Pühl, K., 1997: Identitätspolitik und die Transformation von Staatlichkeit: Geschlechterverhältnisse und Staat als komplexe materielle Relation, in: Politische Vierteljahresschrift, Sonderheft, Kreisky, E., Sauer, B., (Hrsg), Geschlechterverhältnisse im Kontext politischer Transformation, 28: 220-240.

Die Gruppe von Lissabon, 1997: Grenzen des Wettbewerbs, Luchterhand.

Elson, D., 1994: Micro, Meso, Macro: Gender and Economic Analysis in the Context of Policy Reform, in: Bakker, I., Strategic Silence. Gender and Economic Policy, Ottowa, Kanada.

Enloe, C., 1989: Bananas, Beaches, and Bases: Making Feminist Sense of International Politics. Berkeley, Kalifornien.

Esser, J., Fach W., 1981: Korporatistische Krisenregulierung im MODELL DEUTSCHLAND, in: Alemann von, U., (Hrsg.), Neokorporatismus. Frankfurt/Main.

Faludi, S., 1991: Backlash. New York.

Fernández Kelly, M.P., 1989: Broadening the Scope: Gender and International Economic Development, in: Sociological Forum, Nr. 4, 11-35.

Fernández Kelly, M.P., Garcia A., 1992: Power Surrendered, Power Restored: The Politics of Home and Work among Hispanic Women in Southern California and Southern Florida, in: Louise Tilly and Patricia Guerin, (Hrsg), Women and Politics in America, New York.

Fernández Kelly, M.P., Sassen S., 1995: Recasting Women in the Global Economy: Internationalization and Changing Definition of Gender, in: Bose, Ch. E., and Acosta-Belén, E., Women in the Latin American Development Process, Philadelphia.

Fraser, N., 1990: Struggle Over Needs: Outline of a Socialist-Feminist Critical Theory of Late-Capitalist Political Culture, in: Gordon, L., Women, the State, and Welfare, Wisconsin.

Friese, M., 1995: Modernisierungsfallen im historischen Prozeß. Zur Entwicklung der Frauenarbeit im gewandelten Europa, in: Berliner Journal für Soziologie, H. 2, S. 149-162.

Giddens, A., 1995: Konsequenzen der Moderne. Frankfurt/Main.

Giddens, A., 1984: The Constitution of Society. Cambridge.

Gordon, L. (Hrsg), 1990: Women, the State, and Welfare, Wisconsin.,

Gordon, L., 1993: Gender, State and Society: A Debate with Theda Skocpol, in: Contention, Vol. 2, No. 3, Spring, 139-155.

Hagen, E., Jenson, J., 1988: Paradoxes and Promises. Work and politics in the postwar years, in: Jenson, J., Hagen, Reddy, C. (Hrsg), Feminization of the Labor Force, New York.

Harvey, D., 1989: The Condition of Post-Modernity, Oxford.

Haraway, D., 1997: Modest_Witness@Second_Millenium.FemaleMan(C)_Meets_OncoMouse ${ }^{\mathrm{TM}}$ New York.

Held, D., 1991: Democracy, the Nation State and the Global System, in: Economy and Society, Vol. 20, No. 2: 138-172.

Hirsch, J., 1995: Der nationale Wettbewerbsstaat. Berlin-Amsterdam.

Hirst, P., Thompson, G., 1996: Globalization in Question, Oxford.

Hossfeld, K., 1990: 'Their Logic against Them': Contradictions in Sex, Race, and Class in Silicon Valley, in: Ward, K., (ed.)., Women Workers and Global Restructuring, Cornell, Ithaca. 
Kerchner, B., Wilde, G., (Hrsg)., 1997: Staat und Privatheit. Opladen.

Kontos, S., 1990: Zum Verhältnis von Autonomie und Partizipation in der Politik der neuen Frauenbewegung, in: Schaeffer-Hegel, B., (Hrsg), Vater Staat und Seine Frauen, Pfaffenweiler.

Kreisky, E., Sauer, B., (Hrsg), 1997: Das geheime Glossar der Politikwissenschaft. Frankfurt/M.

— (Hrsg), 1995: Feministische Standpunkte in der Politikwissenschaft. Frankfurt/M.

Kulawik, T., 1991/92: Autonomous Mothers? West German Feminism Reconsidered, in: German Politics and Society, Issue 24/25: 67-86.

Kulawik, T., Sauer, B., 1996: Der halbierte Staat. Frankfurt/New York.

Kurz-Scherf, I., 1996: Krise der Arbeit - Krise der Gewerkschaften, in: Weibblick, Nr. 25, S. 20-29.

Jessop, B., 1997: Nationalstaat, Globalisierung, Gender, in: Politische Vierteljahreschrift, Sonderheft, Kreisky, E., Sauer, B., (Hrsg.), Geschlechterverhältnisse im Kontext politischer Transformation, 28: 262-292.

- 1994: Veränderte Staatlichkeit, in: Grimm, D., (Hrsg), Staatsaufgaben, Baden-Baden.

- 1990: State Theory, Oxford.

Lang, S., 1997: The NGOization of Feminism, in: Scott, J.W., Kaplan, C., Keates, D. (eds.), Transitions, Environments, Translations, New York and London.

Larner, W., 1996: The 'New Boys': Restructuring in New Zealand, 1984-94, in: Social Politics. Jg. 3, Nr. 1, S. 32-56.

Lemoine, M., 1998: Die Arbeiter Zentralamerikas als Geiseln der 'maquilas', in: Le Monde Diplomatique, Die Tageszeitung, März: 14-15.

Lewis, J., Ostner, I., 1994: Gender and the Evolution of European Social Policies, ZeSArbeitspapier Nr. 4/94, Centre for Social Policy Research, Bremen.

Mahnkopf, B., 1997: Die 'Feminisierung der Beschäftigung' - in Europa und Anderwo, in: Weibblick, H. 718, S. 22-31.

Mann, P.S., 1994: Micro-Politics: Agency in a Postfeminist Era, Minnesota.

Mayntz, R., 1997: Soziale Dynamik und politische Steuerung. Frankfurt a.M.

McDowell, L., 1997: Capital Culture. Gender At Work in the City. Oxford.

McGrew G., Lewis, P., 1992: Globalization and the Nation States, Cambridge.

Messner, Dirk., 1997: Netzwerktheorien: Die Suche nach Ursachen und Auswegen aus der Krise staatlicher Steuerungsunfähigkeit, in: Altvater, E., Brunnengräber, A., Haake, M., Walk, H., Vernetzt und verstrickt, Münster, S. 7-64.

Pateman, C., 1988: The Sexual Contract, Stanford.

Polanyi, K., 1957: The Great Transformation., Boston.

Rowbotham, S., Mitter, S., 1994: Dignity and Daily Bread, New York.

Sassen, S., 1996: Toward a Feminist Analytics of the Global Economy, in: Indiana Journal of Global Legal Studies, Vol. 4 (Fall): S. 7-41.

Sassoon, A.S., 1987: Women and the State, London and New York.

Schmitter, P.C., 1979: Still the Century of Corporatism? In: Schmitter, P., Lehmbruch, G., (Hrsg.), Trends Toward Corporatist Intermediation. Beverly Hills, California.

Smith, J., Wallerstein, I., 1992: Creating and Transforming Households. The Constraints of the World-Economy. Cambridge.

Stetson, D.M., Mazur, A., (eds.), 1995:Comparative State Feminism, Thousand Oaks.

Strange, S. 1996: The Retreat of the State, Cambridge.

Thurow, L.S., 1997: The Future of Capitalism, New York.

Ward, K., (ed.) 1990: Women Workers and Global Restructuring. Ithaca.

Ward, K., Pyle J.L., 1995: Gender, Industrialization, Transnational Corporations, and Development: An Overview of Trends and Patterns, in: Bose, C.E., Acosta-Belen, E., Women in the Latin American Development Process, Philadelphia.

Wichterich, Ch., 1998: Die globalisierte Frau. Hamburg.

Young, B., 1998: Triumph of the Fatherland: German Unification and the Marginalization of Women, Michigan.

- 1997: Politik und Ökonomie im Kontext von Globalisierung, in: Politische Vierteljahresschrift, Sonderheft, (Hrsg), Kreisky, E., Sauer, B., Geschlechterverhältnisse im Kontext po- 
litischer Transformation. 28, S. 137-151. 